\title{
Defining the broader, medium and narrow autism phenotype among parents using the Autism Spectrum Quotient (AQ)
}

\author{
Sally Wheelwright*, Bonnie Auyeung, Carrie Allison and Simon Baron-Cohen*
}

\begin{abstract}
Background: The Autism Spectrum Quotient (AQ) is a self-report questionnaire for quantifying autistic traits. This study tests whether the AQ can differentiate between parents of children with an autism spectrum condition (ASC) and control parents. In this paper, the use of the AQ to define the broader, medium and narrow autism phenotypes (BAP, MAP, NAP) is reported, and the proportion of parents with each phenotype is compared between the two groups.

Methods: A sample of 571 fathers and 1429 mothers of children with an ASC completed the AQ, along with 349 fathers and 658 mothers of developing typically children.

Results: Both mothers and fathers of the diagnosed children scored higher than the control parents on total AQ score and on four out of five of the subscales. Additionally, there were more parents of diagnosed children with a BAP, MAP or NAP.

Conclusions: The AQ provides an efficient method for quantifying where an individual lies along the dimension of autistic traits, and extends the notion of a broader phenotype among first-degree relatives of those with ASC. The AQ is likely to have many applications, including population and clinical screening, and stratification in genetic studies.
\end{abstract}

\section{Background}

Autism spectrum conditions (ASC) are diagnosed on the basis of behaviour, specifically difficulties in social and communication development, alongside repetitive behaviour and unusually narrow strong interests [1]. The evidence for the genetic basis of ASC initially came from twin studies of classic autism $[2,3]$ and more recently twin studies of autistic traits [4-6]. Progress from these epidemiological findings to identifying specific DNA sequence variations that cause ASC has been slow: replication of results has been hampered by methodological issues such as limited power, varying designs and genotyping, along with imprecise phenotypic definitions [7]. Another reason for limited progress is that although ASC has a high inheritance rate, it is genetically heterogeneous. Rare de novo mutations and chromosomal abnormalities could account for as many as $20 \%$ of ASC cases, but common allelic variation is also important, suggesting that a cate-

* Correspondence: sjw18@cam.ac.uk, sb205@cam.ac.uk

Autism Research Centre, Department of Psychiatry, University of Cambridge, Douglas House, 18b Trumpington Rd, Cambridge, CB2 8AH, UK

Full list of author information is available at the end of the article gorical approach to case ascertainment may not always be the best approach [8].

Indeed, a case-control or categorical approach to diagnosis ignores the view that autism is not just a spectrum within the clinical population, but that autistic traits are continuously distributed right through the general population $[5,9]$. Taking seriously the dimensional view necessitates the use of techniques such as quantitative trait loci (QTL) analysis. Quantitative traits are characteristics that can be associated with a particular condition but are also continuously distributed in the non-affected population. If QTL is to be a successful tool in the search for autism susceptibility genes, then an instrument that can quantify autism traits in both affected and non-affected individuals is required. As well as quantifying autism traits within the whole population, such an instrument could also be used to define the broader autism phenotype (BAP).

The BAP is generally considered to be a subclinical set of characteristics or traits that index familiality and/or genetic liability to autism. This conception holds that the BAP is milder but qualitatively similar to the diagnosed 
autism phenotype. BAP characteristics were first observed by Kanner [10], and were also noted in Folstein and Rutter's early twin study [3], which found a higher concordance rate for a more broadly defined cognitive impairment.

Given the increasing importance of measuring the BAP, not only in genetic studies, but across autism research (for example, in neuroimaging and cognitive studies), it is important to select the right tool. There are already at least four instruments available for the assessment of the BAP: the Family History Interview (FHI), developed by Rutter and Folstein; the Social Responsiveness Scale (SRS) [11]; the Broader Phenotype Autism Symptom Scale (BPASS) [12]; and the Broad Autism Phenotype Questionnaire (BAPQ) [13].

The FHI measures autism-related traits in family members via interview with one member. The instrument covers social and communication skills and range of interests. An algorithm determines the presence or absence of the BAP in the three domains. The FHI was used by Bolton and colleagues [14] to demonstrate higher rates of the BAP in the parents and siblings of probands with autism compared with probands with Down's syndrome. This finding was replicated by Piven and colleagues [15].

The SRS is a 65-item questionnaire, which is completed by an adult informant. Its focus is on the ability of the subject of the questionnaire to engage in emotionally appropriate reciprocal social interaction and communication. Using the SRS, a higher number of autistic traits have been observed in the siblings of children with autism [16].

The BPASS was developed specifically for use in QTL studies [12]. This instrument comprises seven semistructured interview items and six observation items, which together cover social motivation, social expressiveness, conversational skills and repetitive/restricted behaviours. The BPASS is for use with both adults and children.

The most recently published instrument, the BAPQ, comprises 36 items, 12 in each of three subscales: aloof personality, pragmatic language deficits, and rigid personality. Ideally, scores from self- and informant reports are combined to give a best-estimate rating. The BAPQ has been validated using direct clinical assessment of BAP [13].

We suggest that when choosing an instrument to measure autistic traits in both the affected and non-affected populations, the following qualities are important. (i) A good BAP measure should be quantitative, with a wide range of possible scores, avoiding both ceiling and floor effects. (ii) The content validity should be such that the instrument can distinguish people with from those without an ASC. (iii) Versions of the same instrument should be available for use with individuals of different ages, both adults and children. (iv) Ideally, the instrument should produce a normal or near-normal distribution in the general population. (v) The instrument should have good test-retest reliability. (vi) It should have good crosscultural applicability. (vii) It should correlate with either biological and/or psychological measures of the broader phenotype (concurrent validity). Finally, (viii) it should be quick and easy to use: participants in research projects should be subjected to the least amount of testing possible, without compromising the validity of the research project.

Bearing in mind these qualities, each of the four instruments described above have some weaknesses: the FHI and BPASS are not truly quantitative and are both timeconsuming to administer, the SRS is not normally distributed, and the BAPQ is currently limited to adults.

In this paper, we propose that an alternative to the four instruments already available is the Autism Spectrum Quotient (AQ) [9]. The AQ was developed to assess where an individual lies on the autism spectrum (that is, how many autistic traits that person exhibits). Originally designed for adults, there are now child ( 4-11 years old) [17] and adolescent (12-16 years old) [18] versions available (both completed by parents to improve accuracy). The questionnaires are freely available to download http:/ /www.autismresearchcentre.com.

The AQ for adults has the format of a self-report, forced-choice questionnaire and is at the reading level of a typical 10 -year-oldl. It can be used by adults with an IQ in the average range, who can read and understand at least to this level. There are 50 items, covering behaviours across five domains: communication, social skills, attention switching, imagination, and attention to detail. Each item has the format of a statement with which the respondent rates how strongly they agree or disagree, using a four-point scale. In fact, each item is only scored as ' 1 ' if the person reports the autistic trait, and ' 0 ' if they do not, so total scores on the AQ can range from 0 to 50 . Some researchers have used a four-point Likert scale for the AQ [19-21], but this does not significantly alter the pattern of results. The AQ is quick and easy to use and produces a near-normal distribution in the general population [9].

The adult AQ has been used extensively, and has been shown to have consistent results both across time [22] and culture [20,23]. The AQ demonstrates high heritability [5]. The AQ score is a good predictor of clinical diagnosis [24], correlates with brain function [25], single nucleotide polymorphisms (SNPs) in candidate genes [26], social attention $[27,28]$ and even prenatal testosterone levels [29].

A previous study, by Dorothy Bishop and colleagues, suggested that AQ scores differentiate parents of children with an ASC from control parents on two subdomains: 
communication and social skills [29]. Although this was a pioneering study, its limitation was that it only tested 65 mothers and 46 fathers of children with a pervasive developmental disorder (PDD) diagnosis, and 48 mothers and 37 fathers of control children. Although this study indicates the potential of the AQ to serve as a measure of the BAP, it is not clear whether the lack of significance on three of the five subdomains of the $A Q$ is because these play no role in the BAP or because the sample size was too small. Therefore, in the current study, we used the AQ with a much larger sample of parents.

Our first aim was thus to test if the results found by Bishop and colleagues [29]could be replicated in a new, and considerably larger, sample. The second aim was to test the proportion of parents of children with an ASC who demonstrate the BAP, the medium autism phenotype (MAP) and the narrow autism phenotype (NAP). The MAP and NAP are new concepts. An individual with the NAP has a large number of autistic traits, and most (but not all) people with the NAP will also have a diagnosis on the autism spectrum. Not all individuals with the NAP will be diagnosed because there are many reasons why an individual may seek or receive a diagnosis: the NAP is one band within the phenotypic spectrum and does not take into consideration the individual's context, which may mediate whether they actually need or receive a diagnosis. Using the $\mathrm{AQ}$, we define the NAP as those scoring $\geq 3$ standard deviations (SDs) above the mean. An individual with the MAP has a medium number of autistic traits (defined as individuals scoring between 2 to 3 SDs above the mean on the AQ), and an individual with the BAP has even fewer traits, but still significantly more than average (defined as individuals scoring 1 to 2 SDs above the mean on the AQ). People with the MAP or BAP are unlikely to require clinical intervention, but their phenotypic status may be informative at a genetic level.

\section{Methods}

\section{Instrument}

Full details about the construction of the $A Q$ are available elsewhere [9]. The AQ consists of a series of 50 statements. Participants are asked to indicate whether they 'strongly agree', 'slightly agree', 'slightly disagree' or 'strongly disagree' with each statement. Each item scores zero or one, with one point being awarded if the participant chooses the 'autistic trait' response. On half the items, the 'autistic trait' response is 'slightly/strongly agree', and on half the items the 'autistic trait' response is 'slightly/strongly disagree'. A total score is calculated by summing across items. The BAP, MAP and NAP scores are calculated using the mean and SD from the combined male and female control individuals shown in Table $1(\mathrm{n}=$ 1761 , mean $\pm \mathrm{SD}=16.3 \pm 5.9$; taken from [22]) $\mathrm{BAP}$ is defined as AQ scores of 1 to 2 SDs above the mean (AQ scores of 23 to 28). MAP is defined as AQ scores of 2 to 3 SDs above the mean (AQ scores of 29 to 34). NAP is defined as $\mathrm{AQ}$ scores $\geq 3$ SDs above the mean (AQ scores of $35+$ ).

\section{Participants and procedure}

Two groups of parents took part in the study: parents of children with a diagnosis on the autism spectrum and parents of families who only had children who were developing typically. The parents of children with an ASC were recruited from families who registered on the Cambridge University Autism Research Centre volunteer database between 2002 and 2009 (online at http:// www.autismresearchcentre.com). Ethics approval for the database and questionnaire collection was provided by the Cambridge Psychology Research Ethics Committee. Parents gave informed consent to take part in the study electronically.

Parents registering on the database are asked to state the diagnosis of their child, who made the diagnosis, and where and when. In addition, parents are asked to provide information about the IQ and language development of their child, although this is not obligatory. After registering, the parents are invited to complete an $\mathrm{AQ}$, and are asked to encourage the other biological parent to also register and complete an AQ. The advantage of using an online website to collect these data is that large samples can be collected. The disadvantage is that diagnosis cannot be validated in every case. All parents included in this study reported that their children had received their diagnosis from experienced clinicians in recognized clinics and according to the criteria from the Diagnostic and Statistical Manual of Mental Disorders, 4th edition (DSM-IV) or the International Statistical Classification of Diseases and Related Health Problems, 10th Revision (ICD-10).

In total, 1582 families took part, with 571 fathers and 1429 mothers completing the AQ. There were questionnaires from 418 couples. The mean age of the fathers for whom these data were available $(n=551)$ was $44.0 \pm 6.9$ years and for mothers $(n=1228)$ was $41.2 \pm 7.5$ years. The numbers of diagnosed and developing typically children in the 1582 families are shown in Tables 1 and 2. There were 1752 children with a diagnosis of ASC:1472 male and 280 female, giving a male:female ratio of 5.3:1. of he 1752 children, 727 were reported to have autism, 725 to have Asperger's syndrome (AS), 185 to have high functioning autism (HFA) and 115 to have pervasive development disorder. As a further check on diagnostic subtype, the registration process asks about comorbid language delays or learning difficulties. A diagnosis of AS is only registered if the parent indicates that the child did not have any language or general developmental delay. A diagnosis of HFA is only registered if the parent indicates 
Table 1: Number of children developing typically in the 1582 ASC families

\begin{tabular}{llllll}
\hline Number of siblings developing typically & 0 & 1 & 2 & 4 & 5 \\
\hline Number of families & 1260 & 217 & 82 & 14 & 8 \\
\hline ASC $=$ autism spectrum condition. & & &
\end{tabular}

that there was no general developmental delay even if language was delayed.

For the control group, the AQ was sent to the parents of 1255 children ( 633 girls, 622 boys) who were participating in a large epidemiological study of social and communication skills in primary school-age children [30-32]. This part of the project was ethically approved by the National Health Service Suffolk Research Ethics Committee, and written informed consent was obtained from participating parents. Two copies of the questionnaire and two consent forms were posted to each family: one set for the mother and one set for the father. A post-paid envelope was included for participants to return the questionnaires and consent forms. This sample was originally ascertained by inviting 136 mainstream primary schools in Cambridge City, east and south Cambridgeshire, and Fenland in the UK to participate in research $(\mathrm{n}=92(68 \%)$, agreed). In total, 1012 questionnaires were received from 669 families $(661$ from mothers, 351 from fathers) with 343 pairs, a $40 \%$ response rate. Two families were excluded because they reported a child with a diagnosis or suspected diagnosis on the autism spectrum. One family was excluded because they reported a child with Down's syndrome. Questionnaires from 666 families (558 mothers and 349 fathers) with 341 pairs were analysed. The mean age (at the time of completing the $A Q)$ of the fathers for whom these data were available $(\mathrm{n}=344)$ was $44.6 \pm 5.0$ years and for mothers $(\mathrm{n}$ $=644)$ was $42.4 \pm 4.7$. The children in these families were, according to parental report, all developing typically, and the numbers are shown in Table 3; in total, there were 1532 children in these families.

\section{Results}

The mean AQ scores and standard deviations are shown for the two groups in Table 4, along with control data and AS data [22] for comparison. The distribution of scores in the two parent groups are shown in Figure 1 and Figure 2.

Table 2: Number of children with a diagnosis in the 1582 ASC families

\begin{tabular}{lllll}
\hline Number of diagnosed children & 1 & 2 & 3 & 4 \\
\hline Number of families & 1423 & 150 & 7 & 2 \\
\hline ASC
\end{tabular}

ASC $=$ autism spectrum condition.
Analysis of variance (ANOVA), with between-subject factors of 'group' (ASC parents versus control parents) and 'gender' was carried out on total AQ score. There was a significant main effect of group $(\mathrm{F}(1,3003)=45.8, \mathrm{P}<$ 0.001 ), with the ASC parents scoring higher than the control parents, and of gender $(\mathrm{F}(1,3003))=104.3, \mathrm{P}<0.001)$, with males scoring higher than females. Although the group $\times$ gender interaction was significant $(F(1,3003)=$ $6.0, \mathrm{P}<0.05)$, simple effect tests indicated that the gender difference held up in both groups separately (ASC parents: $F(1,3003)=42.0, P<0.001$; control parents: $\mathrm{FF}(1,3003)=62.5, \mathrm{P}<0.001)$ and that the group difference also held up when each gender was tested separately $($ males: $\mathrm{F}(1,3003)=6.9, \mathrm{P}<0.01$; females: $\mathrm{F}(1,3003))=$ 65.4, $\mathrm{P}<0.001)$. The effect sizes for the group, gender, and group $\times$ gender interaction were all small: partial eta squared $(\eta 2)$ values were $0.02,0.03$ and 0.002 respectively, indicating that these factors accounted for just a small amount of the overall variance.

Table 5 shows the means and standard deviations of the five subdomains of the $\mathrm{AQ}$ in the parent groups only. Also shown are the $F$ values and partial $\eta^{2}$ values generated by separate ANOVAs, with between-subject factors of group (ASC parents versus control parents) and gender, for each of the five subdomains. Inspection of the means suggests that for each subcategory, males scored higher than females, and the ASC parents scored higher than the control parents. The exception to this was the subcategory 'attention to details'; in this, there was no sex difference and the control parents scored higher than the ASC parents. These observations were partly confirmed by the ANOVAs and simple effect tests. Similar to the total AQ score, the subcategories 'attention switching' and 'imagination' had significant main effects of group and gender and a significant group $\times$ gender interaction. Also similar to the total AQ score, simple effect tests indicated that for both subcategories the sex difference held up in both groups separately, and that the group difference also held up when each sex was tested separately $(P$ $<0.01$ for all). The category 'communication and social skills' both had significant main effects of group and gender, but no interaction between them, whereas the subcategory 'attention to detail' only had a significant main effect of group. Again, all the effect sizes are small.

The number of parents matching each of the described phenotypes (the BAP, MAP and NAP) was computed 
Table 3: Number of children in the 666 control families

\begin{tabular}{llllllll}
\hline Number of children & 1 & 2 & 3 & 4 & 5 & 6 & 7 \\
\hline Number of families & 85 & 351 & 183 & 41 & 5 & 0 & 1
\end{tabular}

along with the number scoring in the average range (within $1 \mathrm{SD}$ of the mean) and the low range $(<1 \mathrm{SD}$ below the mean). These data are presented in Figure 2.

Examination of Figure 3 shows that ASC fathers had the highest percentage of scorers across the BAP, MAP and NAP range (approximately 33\%), compared with $23 \%$ of ASC mothers, $22 \%$ of control fathers and $9 \%$ of control mothers. The finding that a similar proportion of ASC mothers and control fathers scored in the BAP or higher range is consistent with the 'extreme male brain' theory of autism [33]. More ASC mothers scored in the MAP and NAP range than control fathers $\left(X^{2}=8.2\right.$, degree of freedom (d.f.) $=1, P<0.01)$. Control mothers had the lowest proportion in the BAP, MAP and NAP ranges, and the fewest number scoring in at least the BAP range. About $50 \%$ of the ASC parents and control mothers score in the average range, compared with about $60 \%$ of the control fathers. Control mothers were over-represented in the low range compared with ASC mothers $\left(X^{2}=11.2\right.$, d.f. $=1$, $P<0.01)$. There was no difference between the two groups in the proportion of fathers scoring in the low range $\left(\chi^{2}=1.7\right.$, d.f. $\left.=1, P>0.05\right)$.

\section{Discussion}

The AQ was developed almost 10 years ago as an instrument to rapidly quantify autistic traits in both clinical and non-clinical samples. Evidence for the construct validity of the AQ and the use of a self-report instrument for quantifying autistic traits comes from a wide variety of autism-related studies in which AQ score correlates with outcome [24-26,34-42]. The AQ is thus a reasonable tool for assessing whether individuals can be classified as having the BAP. Measuring the BAP is increasingly important in genetic studies, and is also important in describing participants in other types of research. Although it may seem contradictory to be employing cut-off points on a scale that was devised precisely to avoid categorical distinctions, other psychometric measures (such as IQ) have long used this approach because there may be predictive value in subgrouping by severity. We thus also introduce the terms MAP and NAP as additional subgroup classifications, while acknowledging that the cut-off points are purely statistically defined (by number of SDs above the mean) rather than 'carving Nature at its joints'.

In the present sample, parents of children with ASC scored significantly higher on the AQ than control parents, both on total AQ score and on four of five of the subcategories. This replicates and extends the earlier finding by Bishop and colleagues [29] in a much larger sample.

The effect sizes were all small, which is not surprising, as we would not predict the BAP to be present in all parents. Comparing the differences between parents who do and do not show the BAP will be important in future research. Furthermore, our sample is heterogeneous; it includes parents of children with different diagnoses on the autism spectrum, some of whom come from multiplex and some from simplex families. In addition, we found not only are the rates of BAP higher in parents of children with an ASC than in control parents, but the rates of MAP and NAP are also higher, as measured by the AQ. This was true for both mothers and fathers.

Table 4: Mean total AQ scores for the parent groups

\begin{tabular}{|c|c|c|c|c|c|c|}
\hline & \multicolumn{3}{|c|}{ Males } & \multicolumn{3}{|c|}{ Females } \\
\hline & $\mathbf{n}$ & Mean & SD & $\mathbf{n}$ & Mean & SD \\
\hline ASC parents & 571 & 19.2 & 9.5 & 1429 & 16.4 & 9.5 \\
\hline Control parents & 349 & 17.7 & 6.9 & 658 & 13.1 & 6.3 \\
\hline AS group & 69 & 35.9 & 7.8 & 56 & 37.4 & 8.2 \\
\hline Control individuals & 723 & 17.4 & 6.2 & 1038 & 15.5 & 5.6 \\
\hline
\end{tabular}

Data from an AS group and controls, taken from [22] are also shown for reference.

$A Q=$ Autism Spectrum Quotient; ASC = autism spectrum condition; $A S=$ Asperger's syndrome. 


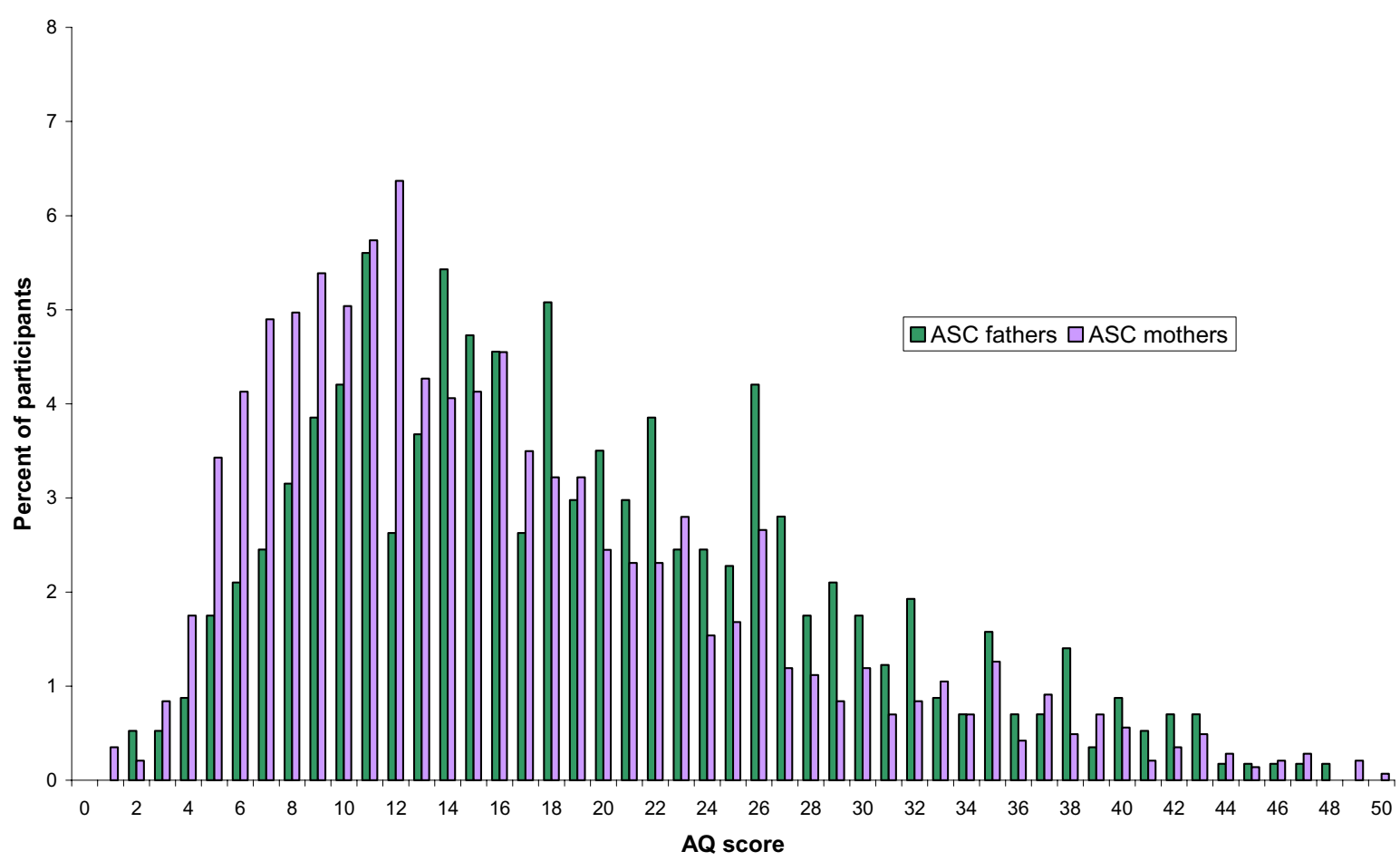

Figure 1 Autism Spectrum Quotient (AQ) scores in the Autism Spectrum Condition (ASC) parent group.

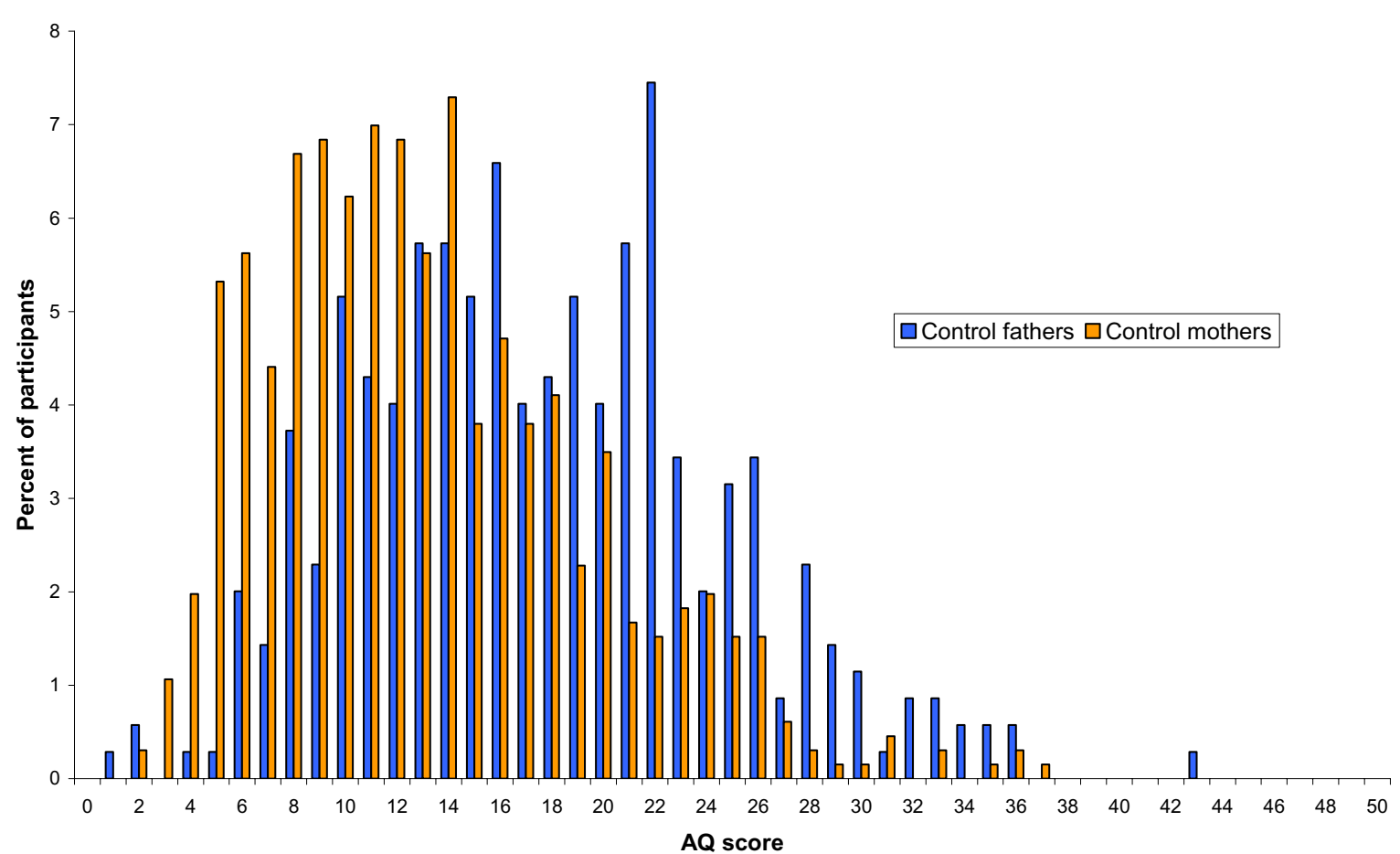

Figure 2 Autism Spectrum Quotient (AQ) scores in the control parent group 
Table 5: Mean (and standard deviation) scores on each of the AQ subcategories in the parent groups

\begin{tabular}{|c|c|c|c|c|c|}
\hline & Communication & Social skills & Attention switching & Imagination & Attention to detail \\
\hline ASC fathers & $3.1(2.4)$ & $4.0(2.9)$ & $4.5(2.6)$ & $3.2(2.3)$ & $4.4(2.3)$ \\
\hline ASC mothers & $2.6(2.4)$ & $3.1(2.8)$ & $4.0(2.5)$ & $2.4(2.2)$ & $4.4(2.3)$ \\
\hline Control fathers & $2.6(1.9)$ & $3.1(2.5)$ & $4.2(2.2)$ & $3.1(2.1)$ & $4.7(2.1)$ \\
\hline Control mothers & $1.7(1.6)$ & $1.9(2.1)$ & $3.1(2.0)$ & $1.8(1.7)$ & $4.7(2.2)$ \\
\hline \multirow{2}{*}{$\begin{array}{l}\text { F value for group main effect (and partial } \\
\text { eta }^{2} \text { ) }\end{array}$} & $55.6^{* *}$ & $91.8^{* *}$ & $39.4^{* *}$ & $18.4^{* *}$ & $11.1^{*}$ \\
\hline & $(0.02)$ & $(0.03)$ & $(0.01)$ & $(0.006)$ & $(0.004)$ \\
\hline \multirow{2}{*}{$\begin{array}{l}\text { F value for sex main effect (and partial } \\
\text { eta }^{2} \text { ) }\end{array}$} & $58.6^{* *}$ & $85.9^{* *}$ & $71.0^{* *}$ & $156.0^{* *}$ & 0.01 \\
\hline & $(0.02)$ & $(0.03)$ & $(0.02)$ & $(0.05)$ & $(0.00)$ \\
\hline \multirow{2}{*}{$\begin{array}{l}F \text { value for group } x \text { sex interaction (and } \\
\text { partial eta }{ }^{2} \text { ) }\end{array}$} & 3.6 & 2.1 & $9.2^{*}$ & $6.9 *$ & 0.01 \\
\hline & $(0.001)$ & $(0.001)$ & $(0.003)$ & $(0.002)$ & $(0.00)$ \\
\hline
\end{tabular}

$F$ values (and partial eta ${ }^{2}$ ) from the ANOVAs are presented.

${ }^{*} P<0.01,{ }^{* *} P<0.001$

$A Q$, Autism Spectrum Quotient; ASC, autism spectrum condition

\section{Study limitations}

There are three methodological limitations with this study. First, information about the families, including child diagnosis, was fully reliant on parental report. Secondly, the questionnaires in the ASC and control groups were collected in slightly different ways. The questionnaires from the ASC group were collected from parents registering an interest in taking part in research, mostly via an online website, although some were paper copies. The questionnaires were collected over a period of 7 years. By contrast, the control group questionnaires were collected by post, over a period of a few weeks. Although we do not think a person's AQ score will be affected by the method of questionnaire administration (because the wording remains identical) this assumption warrants future testing. The time difference between data collection was not ideal, because public awareness of autism and related conditions has increased. However, it is not clear what influence, if any, this would have on individual's response. Future studies should try to ensure that data collection methods are identical for all participants.

Finally, a third limitation is that our control group only comprised families with children who were developing typically. Future studies could also include families who have children with a different developmental disability, to test if there are any effects on how parents report their own characteristics after the diagnosis of a child is made. We doubt this would have contributed to the present results, because the AQ measures a range of domains, including both social and non-social (attentional) characteristics.

\section{Conclusions}

This study provides evidence that the AQ is a useful tool for assessing autism phenotypes in non-clinical samples. The novel terms 'medium autism phenotype' and 'narrow autism phenotype' are introduced to extend the established term 'broader autism phenotype', and precise methods of measuring each are provided. We found that $33 \%$ of fathers of children with ASC and $23 \%$ of mothers scored at or above the BAP cut-off point, with significantly more parents of children with ASC showing MAP and NAP than was seen among control parents. Whether the broad, medium and narrow phenotypes are mirrored by either neural, cognitive, endocrine, proteomic or genetic differences will be of interest to test in the future. 


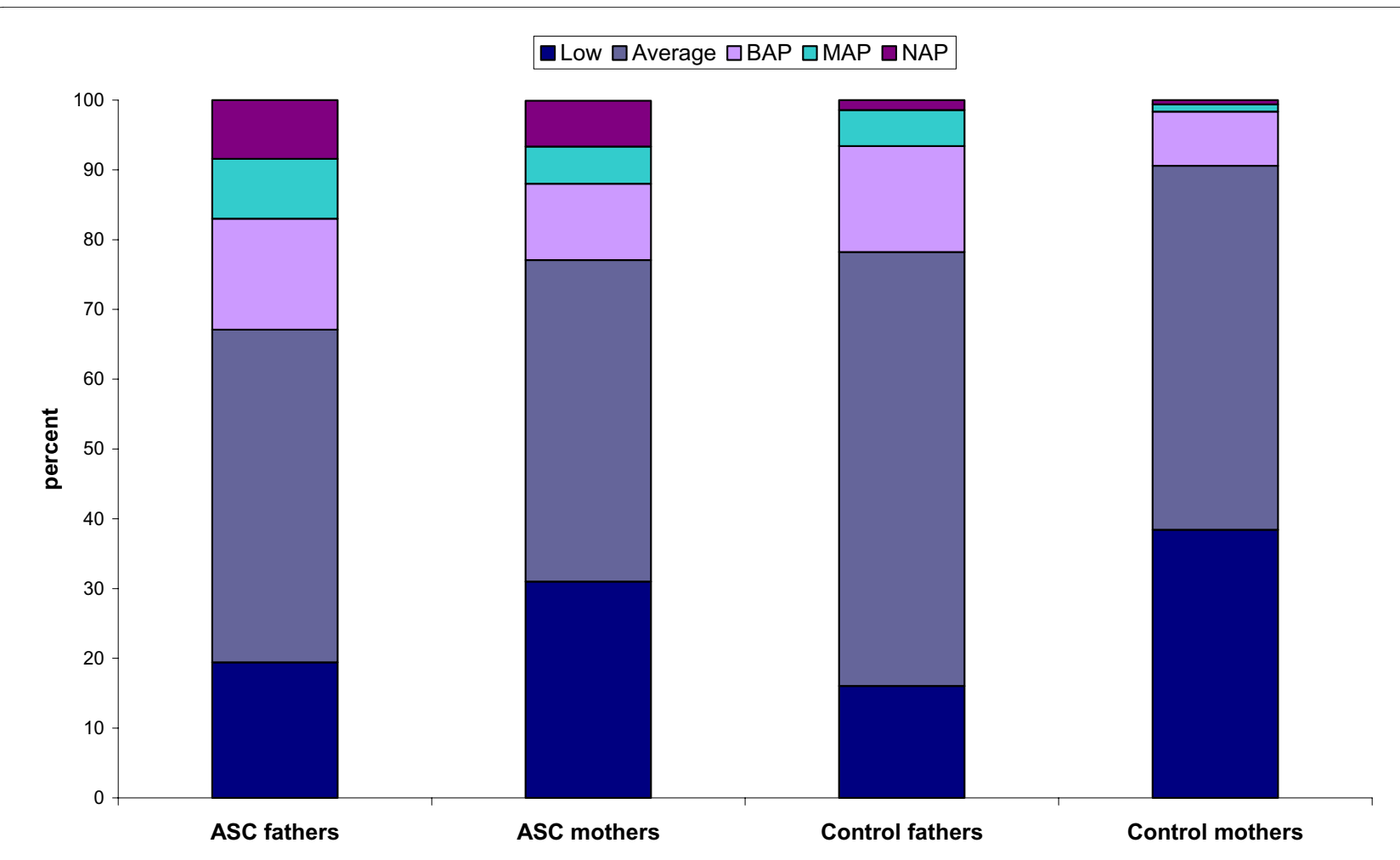

Figure 3 Percent age of parents with each phenotype. ASC = autism spectrum condition; BAP = broader autism phenotype; MAP = medium autism phenotype; NAP = narrow autism phenotype

\section{Competing interests}

The authors declare that they have no competing interests.

\section{Authors' contributions}

All authors read and approved the final manuscript. SW and SBC conceived the study and participated in its design. SW collated the ASC data, performed the statistical analysis and drafted the manuscript. BA and CA collected the control data.

\section{Acknowledgements}

We were supported by grants from the MRC UK, the Shirley Foundation and the Nancy Lurie Marks Family Foundation to SBC during the period of this work. We are grateful to Bhismadev Chakrabarti, Rosa Hoekstra, Edward Sucksmith and Liliana Ruta for valuable discussions. Some data from this work were first presented at the International Meeting for Autism Research (IMFAR), Seattle 2007. This study was conducted in association with the NIHR CLAHRC for NHS Cambridgeshire and Peterborough Foundation Trust. We acknowledge the SCORE project team (Carol Brayne, Patrick Bolton, Fiona Scott, Carol Stott, Jo Williams, Carrie Allison and Simon Baron-Cohen) from which the control group were drawn. Finally, we are indebted to the parents (in both groups) who participated so generously.

\section{Author Details}

Autism Research Centre, Department of Psychiatry, University of Cambridge, Douglas House, 18b Trumpington Rd, Cambridge, CB2 8AH, UK

Received: 5 February 2010 Accepted: 17 June 2010

Published: 17 June 2010

\section{References}

1. A.P.A: DSM-IV Diagnostic and Statistical Manual of Mental Disorders. 4th edition. Washington DC: American Psychiatric Association; 1994
2. Bailey A, Le Couteur A, Gottesman I, Bolton P, Simmonoff E, Yuzda E, Rutter $\mathrm{M}$ : Autism as a strongly genetic disorder: evidence from a British twin study. Psychol Med 1995, 25:63-77.

3. Folstein S, Rutter M: Infantile autism: A genetic study of 21 twin pairs. $J$ Child Psychol Psychiatry 1977, 18:297-321.

4. Constantino JN, Todd RD: Intergenerational transmission of subthreshold autistic traits in the general population. Biol Psychiatry 2005, 57:655-660.

5. Hoekstra RA, Bartels M, Verweij CJH, Boomsma DI: Heritability of autistic traits in the general population. Arch Pediat Adolesc Med 2007, 161:372-377.

6. Ronald A, Happe F, Bolton P, Butcher LM, Price TS, Wheelwright S, BaronCohen S, Plomin R: Genetic heterogeneity between the three components of the autism spectrum: a twin study. J Am Acad Child Adolesc Psychiatry 2006, 45:691-699.

7. Losh M, Sullivan PF, Trembath D, Piven J: Current developments in the genetics of autism: from phenome to genome. J Neuropathol Exp Neurol 2008, 67:829-837.

8. Geschwind DH: Autism: many genes, common pathways? Cell 2008, 135:391-395.

9. Baron-Cohen S, Wheelwright S, Skinner R, Martin J, Clubley E: The AutismSpectrum Quotient (AQ): Evidence from Asperger syndrome/highfunctioning autism, males and females, scientists and mathematicians. J Autism Dev Disord 2001, 31:5-17.

10. Kanner L, Eisenberg L: Early infantile autism, 1943-1955. Psychiatr Res Rep Am Psychiatr Assoc 1957:55-65.

11. Constantino J, N, Przybeck T, Friesen D, Todd R, D: Reciprocal social behaviour in children with and without pervasive developmental disorders. Devl Behav Pediatr 2000, 1:2-11.

12. Dawson G, Estes A, Munson J, Schellenberg G, Bernier R, Abbott R: Quantitative assessment of autism symptom-related traits in probands and parents: Broader Phenotype Autism Symptom Scale. J Autism Dev Disord 2007, 37:523-536. 
13. Hurley RS, Losh M, Parlier M, Reznick JS, Piven J: The broad autism phenotype questionnaire. J Autism Dev Disord 2007, 37:1679-1690.

14. Bolton P, MacDonald H, Pickles A, Rios P, Goode S, Crowson M, Bailey A, Rutter M: A Case-Control Family History Study of Autism. J Child Psychol Psychiatry 1994, 35:877-900.

15. Piven J, Palmer P, Jacobi D, Childress D, Arndt S: Broader autism phenotype: evidence from a family history study of multiple-incidence autism familiies. Am J Psychiatry 1997, 154:185-190.

16. Constantino JN, Lajonchere C, Lutz M, Gray T, Abbacchi A, McKenna K, Singh D, Todd RD: Autistic social impairment in the siblings of children with pervasive developmental disorders. Am J Psychiatry 2006, 163:294-296.

17. Auyeung B, Baron-Cohen S, Wheelwright S, Allison C: The Autism Spectrum Quotient: Children's Version (AQ-Child). J Autism Dev Disord 2008, 38:1230-1240

18. Baron-Cohen S, Hoekstra RA, Knickmeyer R, Wheelwright S: The AutismSpectrum Quotient (AQ) -Adolescent version. J Autism Dev Disord 2006, 36:343-350.

19. Austin EJ: Personality correlates of the broader autism phenotype as assessed by the Autism Spectrum Quotient (AQ). Pers Indiv Differ 2005, 38:451-460

20. Hoekstra RA, Bartels M, Cath DC, Boomsma DI: Factor structure, reliability and criterion validity of the Autism-Spectrum Quotient (AQ): a study in Dutch population and patient groups. J Autism Dev Disord 2008, 38:1555-1566.

21. Stewart ME, Austin EJ: The structure of the Autism-Spectrum Quotient (AQ): Evidence from a student sample in Scotland. Pers Indiv Differ 2009, 47:224-228

22. Wheelwright S, Baron-Cohen S, Goldenfeld N, Delaney J, Fine D, Smith R, Weil L, Wakabayashi A: Predicting Autism Spectrum Quotient (AQ) from the Systemizing Quotient-Revised (SQ-R) and Empathy Quotient (EQ). Brain Res 2006, 1079:47-56.

23. Wakabayashi A, Baron-Cohen S, Wheelwright S, Tojo Y: The AutismSpectrum Quotient (AQ) in Japan: A cross-cultural comparison. J Autism Dev Disord 2006, 36:263-270.

24. Woodbury-Smith MR, Robinson J, Wheelwright S, Baron-Cohen S: Screening adults for Asperger syndrome using the AQ: A preliminary study of its diagnostic validity in clinical practice. J Autism Dev Disord 2005, 35:331-335.

25. Gomot M, Belmonte MK, Bullmore ET, Bernard FA, Baron-Cohen S: Brain hyper-reactivity to auditory novel targets in children with highfunctioning autism. Brain 2008, 131:2479-2488.

26. Chakrabarti B, Dudbridge F, Kent L, Wheelwright S, Hill-Cawthorne G, Allison C, Banerjee-Basu S, Baron-Cohen S: Genes related to sex steroids, neural growth, and social-emotional behavior are associated with autistic traits, empathy, and Asperger syndrome. Autism Res 2009, 2:157-177.

27. Bayliss AP, di Pellegrino G, Tipper SP: Sex differences in eye gaze and symbolic cueing of attention. Q J Exp Psychol A 2005, 58:631-650.

28. Bayliss AP, Tipper SP: Gaze and arrow cueing of attention reveals individual differences along the autism spectrum as a function of target context. Br J Psychol 2005, 96:95-114.

29. Bishop DVM, Maybery M, Maley A, Wong D, Hill W, Hallmayer J: Using selfreport to identify the broad phenotype in parents of children with autistic spectrum disorders: a study using the Autism-Spectrum Quotient. JChild Psychol Psychiatry 2004, 45:1431-1436.

30. Scott F, Baron-Cohen S, Bolton P, Brayne C: Prevalence of autism spectrum conditions in children aged 5-11 years in Cambridgeshire, UK. Autism 2002, 6(3):231-237.

31. Williams J, Scott F, Allison C, Bolton P, Baron- Cohen S, Brayne C: The CAST (Childhood Asperger Syndrome Test): test accuracy. Autism 2005:45-68.

32. Allison C, Williams J, Scott F, Stott C, Bolton P, Baron-Cohen S, Brayne C: The Childhood Asperger Syndrome Test (CAST): test-retest reliability in a high scoring sample. Autism 2007, 11:173-185.

33. Baron-Cohen S: The extreme male brain theory of autism. Trends in Cogn Sci 2002, 6:248-254.

34. Auyeung B, Baron-Cohen S, Ashwin E, Knickmeyer R, Taylor K, Hackett G: Fetal testosterone and autistic traits. Br J Psychol 2009, 100:1-22.

35. Baron-Cohen S, Wheelwright S: The Friendship Questionnaire: An investigation of adults with Asperger syndrome or high-functioning autism, and normal sex differences. J Autism Dev Disord 2003, 33:509-517.
36. Baron-Cohen S, Wheelwright S, Hill J, Raste Y, Plumb I: The 'Reading the Mind in the Eyes' test revised version: A study with normal adults, and adults with Asperger Syndrome or High-Functioning autism. J Child Psychol Psychiatry 2001, 42:241-252.

37. Golan O, Baron-Cohen S, Hill J: The Cambridge Mindreading (CAM) FaceVoice Battery: Testing complex emotion recognition in adults with and without Asperger syndrome. J Autism Dev Disord 2006, 36:169-183.

38. Grinter EJ, Maybery MT, Van Beek PL, Pellicano E, Badcock JC, Badcock DR: Global Visual Processing and Self-Rated Autistic-like Traits. J Autism Dev Disord 2009, 39:1278-1290.

39. Grinter EJ, Van Beek PL, Maybery MT, Badcock DR: Brief Report: Visuospatial Analysis and Self-Rated Autistic-Like Traits. J Autism Dev Disord 2009, 39:670-677.

40. Hermans EJ, van Wingen G, Bos PA, Putman P, van Honk J: Reduced spontaneous facial mimicry in women with autistic traits. Biol Psychol 2009, 80:348-353.

41. Stewart ME, Ota M: Lexical effects on speech perception in individuals with "autistic" traits. Cognition 2008, 109:157-162.

42. Stewart ME, Watson J, Allcock AJ, Yaqoob T: Autistic traits predict performance on the block design. Autism 2009, 13:133-142.

doi: 10.1186/2040-2392-1-10

Cite this article as: Wheelwright et al., Defining the broader, medium and narrow autism phenotype among parents using the Autism Spectrum Quotient (AQ) Molecular Autism 2010, 1:10

\section{Submit your next manuscript to BioMed Central and take full advantage of:}

- Convenient online submission

- Thorough peer review

- No space constraints or color figure charges

- Immediate publication on acceptance

- Inclusion in PubMed, CAS, Scopus and Google Scholar

- Research which is freely available for redistribution

Submit your manuscript at www.biomedcentral.com/submit
C) Biomed Central 